GLASNIK MATEMATIČKI

Vol. 41(61)(2006), $335-343$

\title{
ON THE $n$-FOLD HYPERSPACE SUSPENSION OF CONTINUA, II
}

\author{
Sergio Macías \\ Universidad Nacional Autónoma de México, México \\ In Memoriam Alfredo Macías
}

\begin{abstract}
We continue our study of $n$-fold hyperspace suspensions. We show that $n$-fold hyperspace suspensions of contractible continua are contractible. We prove that $n$-fold hyperspace suspensions are zerodimensional aposyndetic. We also show that hereditarily indecomposable continua have unique $n$-fold hyperspace suspensions.
\end{abstract}

\section{INTRODUCTION}

The notion of $n$-fold hyperspace suspension was introduced in [12]. This concept is a natural extension of the notion of hyperspace suspension introduced by Nadler [18].

Our purpose is to continue the study of the properties of the $n$-fold hyperspace suspensions. We present two results on hyperspaces, namely, Theorem 3.1, Theorem 3.2 which are of interest. Our main results are Theorem 5.1, Theorem 6.1 and Theorem 7.1. Other results such as Theorem 4.1, Theorem 4.3 and Theorem 4.4 are of independent interest.

\section{Definitions}

If $(Z, d)$ is a metric space, then given $A \subset Z$ and $\varepsilon>0$, the open ball about $A$ of radius $\varepsilon$ is denoted by $\mathcal{V}_{\varepsilon}^{d}(A)$, the interior of $A$ is denoted by $\operatorname{Int}_{Z}(A)$, and the closure of $A$ is denoted by $C l_{Z}(A)$. A map means a continuous function.

2000 Mathematics Subject Classification. 54B20.

Key words and phrases. Absolute retract, continuum, $n$-fold hyperspace, $n$-fold hyperspace suspension, retract, strong deformation retract, $Z$-set.

This research was partially supported by the grant 42602 of CONACyT. 
A continuum is a nonempty compact, connected metric space. A subcontinuum is a continuum contained in a space $Z$. A continuum $X$ is said to be indecomposable provided that it cannot be written as the union of two of its proper subcontinua. A continuum is hereditarily indecomposable if all of its subcontinua are indecomposable.

A subcontinnuum $A$ is a retract of the continuum $X$ provided that there exists a map $r: X \rightarrow A$ such that $r(a)=a$ for each $a \in A$, the map $r$ is called a retraction. The subcontinuum $A$ is said to be a strong deformation retract of the continuum $X$ provided that there exists a map $H: X \times[0,1] \rightarrow X$ such that $H(x, 0)=x, H(x, 1)=r(x)$ and $H(a, t)=a$ for each $x \in X$, each $a \in A$ and each $t \in[0,1]$, where $r$ is a retraction, the map $H$ is called a strong deformation retraction.

A subcontinuum $A$ of a continuum $X$ is said to be a $Z$-set if for every $\varepsilon>0$, there exists a map $f: X \rightarrow(X \backslash A)$ such that $d(x, f(x))<\varepsilon$ for each $x \in X$.

An arc is any space homeomorphic to $[0,1]$. The symbol $\mathcal{Q}$ denotes the Hilbert cube.

Given a continuum $X$ we consider the following hyperspaces:

$$
2^{X}=\{A \subset X \mid A \text { is nonempty and closed }\}
$$

and

$$
\mathcal{C}_{n}(X)=\left\{A \in 2^{X} \mid A \text { has at most } n \text { components }\right\},
$$

where $n$ is a positive integer. $\mathcal{C}_{n}(X)$ is called the $n$-fold hyperspace of $X$. These spaces are topologized with the Hausdorff metric defined as follows:

$$
\mathcal{H}_{X}(A, B)=\inf \left\{\varepsilon>0 \mid A \subset \mathcal{V}_{\varepsilon}^{d}(B) \text { and } B \subset \mathcal{V}_{\varepsilon}^{d}(A)\right\},
$$

$\mathcal{H}_{X}$ always denotes the Hausdorff metric on the $n$-fold hyperspace of a continuum $X$. When $n=1$, we write $\mathcal{C}(X)$ instead of $\mathcal{C}_{1}(X)$.

The symbol $\mathcal{F}_{n}(X)$ denotes the $n$-fold symmetric product of $X$; that is:

$$
\mathcal{F}_{n}(X)=\left\{A \in \mathcal{C}_{n}(X) \mid A \text { has at most } n \text { points }\right\} .
$$

Note that, by definition, $\mathcal{F}_{n}(X) \subset \mathcal{C}_{n}(X)$. It is known that $\mathcal{C}_{n}(X)$ is an arcwise connected continuum (for $n=1$, see [17, (1.12)]; for $n \geq 2$, see [10, $3.1])$.

An order arc in $\mathcal{C}_{n}(X)$ is an arc $\alpha:[0,1] \rightarrow \mathcal{C}_{n}(X)$ such that if $0 \leq s<$ $t \leq 1$ then $\alpha(s) \subset \alpha(t)$ and $\alpha(s) \neq \alpha(t)$.

By the $n$-fold hyperspace suspension of a continuum $X$, which is denoted by $H S_{n}(X)$, we mean the quotient space:

$$
H S_{n}(X)=\mathcal{C}_{n}(X) / \mathcal{F}_{n}(X)
$$

with the quotient topology. The fact that $H S_{n}(X)$ is a continuum follows from $[19,3.10]$. Notice that $H S_{1}(X)$ corresponds to the hyperspace suspension $H S(X)$ defined by Nadler in [18]. 
Notation 2.1. Given a continuum $X, q_{X}^{n}: \mathcal{C}_{n}(X) \rightarrow H S_{n}(X)$ denotes the quotient map. Also, let $F_{X}^{n}$ denote the point $q_{X}^{n}\left(\mathcal{F}_{n}(X)\right)$.

Remark 2.2. Note that the sets $H S_{n}(X) \backslash\left\{F_{X}^{n}\right\}$ and $H S_{n}(X) \backslash$ $\left\{q_{X}^{n}(X), F_{X}^{n}\right\}$ are homeomorphic to $\mathcal{C}_{n}(X) \backslash \mathcal{F}_{n}(X)$ and $\mathcal{C}_{n}(X) \backslash\left(\{X\} \cup \mathcal{F}_{n}(X)\right)$, respectively, using the appropriate restriction of $q_{X}^{n}$.

\section{A RESUlt OF $n$-FOLD HYPERSPACES}

In $[11,4.13]$ it was shown that for a continuum $X$ which is an absolute retract, $\mathcal{F}_{1}(X)$ is a strong deformation retract of $\mathcal{C}_{n}(X)$. We prove, under the same hypothesis, that $\mathcal{F}_{n}(X)$ is a strong deformation retract of $\mathcal{C}_{n}(X)$.

A continuum $X$ is said to be an absolute retract provided that for any metric space $Z$ and any embedding $h: X \rightarrow Z, h(X)$ is a retract of $Z$.

The proof of the following theorem is similar to the proof of $[7,2.5]$. We include the details for the convenience of the reader.

Theorem 3.1. If $X$ is an absolute retract, then $\mathcal{F}_{n}(X)$ is a strong deformation retract of $2^{X}$.

Proof. Since $X$ is an absolute retract, $\mathcal{F}_{n}(X)$ is an absolute retract [6, p. 316]. Hence, $\mathcal{F}_{n}(X) \times \mathcal{Q}$ is homeomorphic to $\mathcal{Q}$ [2, 44.1 and 22.1]. Let $q_{0} \in \mathcal{Q}$. Since $\left\{q_{0}\right\}$ is a $Z$-set in $\mathcal{Q}$ [2, (ii), p. 2], it follows that $\mathcal{F}_{n}(X) \times\left\{q_{0}\right\}$ is a $Z$-set in $\mathcal{F}_{n}(X) \times \mathcal{Q}$, and since $\left\{q_{0}\right\}$ is a strong deformation retract of $\mathcal{Q}$, it follows that $\mathcal{F}_{n}(X) \times\left\{q_{0}\right\}$ is a strong deformation retract of $\mathcal{F}_{n}(X) \times \mathcal{Q}$. Let $h:\left(\mathcal{F}_{n}(X) \times \mathcal{Q}\right) \times[0,1] \rightarrow \mathcal{F}_{n}(X) \times \mathcal{Q}$ be a strong deformation retraction from $\mathcal{F}_{n}(X) \times \mathcal{Q}$ onto $\mathcal{F}_{n}(X) \times\left\{q_{0}\right\}$. By [17, (16.18)], $\mathcal{C}(X)$ is contractible. Thus, $\mathcal{F}_{1}(X)$ is a $Z$-set in $\mathcal{C}(X)[7,2.4]$. Hence, $\mathcal{F}_{n}(X)$ is a $Z$-set in $2^{X}[14,2.2]$. By $[17,(1.97)], 2^{X}$ is homeomorphic to $\mathcal{Q}$. Let $f: \mathcal{F}_{n}(X) \rightarrow \mathcal{F}_{n}(X) \times\left\{q_{0}\right\}$ be the homeomorphism given by $f(A)=\left(A, q_{0}\right)$. By Anderson's homeomorphism extension theorem $[8,11.9 .1], f$ can be extended to a homeomorphism $F: 2^{X} \rightarrow \mathcal{F}_{n}(X) \times \mathcal{Q}$. It follows that the map

$$
H: 2^{X} \times[0,1] \rightarrow 2^{X}
$$

given by

$$
H(A, t)=F^{-1}(h(F(A), t))
$$

is a strong deformation retraction from $2^{X}$ onto $\mathcal{F}_{n}(X)$.

As a consequence of Theorem 3.1, we have:

Theorem 3.2. If $X$ is an absolute retract and $n$ is a positive integer, then $\mathcal{F}_{n}(X)$ is a strong deformation retraction of $\mathcal{C}_{n}(X)$.

Proof. Since $X$ is an absolute retract, there exists a strong deformation retraction $H: 2^{X} \times[0,1] \rightarrow 2^{X}$ from $2^{X}$ onto $\mathcal{F}_{n}(X)$ by Theorem 3.1.

Since $X$ is locally connected, $\mathcal{C}_{n}(X)$ is an absolute retract [20, Théorème $\left.\mathrm{II}_{m}\right]$. Hence, there exists a retraction $r: 2^{X} \rightarrow \mathcal{C}_{n}(X)$. Thus, the map $r \circ$ 
$\left(\left.H\right|_{\mathcal{C}_{n}(X) \times[0,1]}\right): \mathcal{C}_{n}(X) \times[0,1] \rightarrow \mathcal{C}_{n}(X)$ is a strong deformation retraction from $\mathcal{C}_{n}(X)$ onto $\mathcal{F}_{n}(X)$.

As consequence of Theorem 5.2 and Theorem 3.2 we obtain:

Corollary 3.3. If $X$ is an absolute retract, then $H S_{n}(X)$ is contractible.

Proof. Since $X$ is an absolute retract, $\mathcal{F}_{n}(X)$ is a strong deformation retraction of $\mathcal{C}_{n}(X)$ (Theorem 3.2). Therefore, the result follows from Theorem 5.2.

\section{General properties}

We continue our study of $n$-fold hyperspace suspensions by noting that if $m<n$, then the $m$-fold hyperspace suspension of a continuum may be embedded in its $n$-fold hyperspace suspension.

Theorem 4.1. Let $X$ be a continuum. Let $n$ and $m$ be positive integers such that $n>m$. Then $H S_{m}(X)$ may be embedded in $H S_{n}(X)$.

Proof. Let $i_{m, n}: \mathcal{C}_{m}(X) \rightarrow \mathcal{C}_{n}(X)$ be the inclusion map. Observe that $i_{m, n}$ is a relation preserving map [4, p. 16]. Now let $h_{m, n}: H S_{m}(X) \rightarrow$ $H S_{n}(X)$ be given by

$$
h_{m, n}(\chi)= \begin{cases}F_{X}^{n} & \text { if } \chi=F_{X}^{m} \\ q_{X}^{n}\left(i_{m, n}\left(\left(q_{X}^{m}\right)^{-1}(\chi)\right)\right) & \text { if } \chi \neq F_{X}^{m}\end{cases}
$$

Note that $h_{m, n}$ is continuous by $\left[4,4.3\right.$, p. 126]. It is clear that $h_{m, n}$ is oneto-one. Since the spaces are compacta, $h_{m, n}$ is an embedding. Therefore, $H S_{m}(X)$ may be embedded in $H S_{n}(X)$.

Question 4.1. For what continua $X$ does the natural embedding in the proof of Theorem 4.1 embed $H S_{m}(X)$ as a retract of $H S_{n}(X)$ ? In particular, what about the case when $X$ is $\mathcal{S}^{1}$ ?

More generally, we ask the following:

Question 4.2. For what continua $X$ can $H S_{m}(X)$ be embedded in $H S_{n}(X)$ as a retract $(m<n)$ ?

Remark 4.2. Let us note that a positive answer to Question 4.1 when $X$ is $\mathcal{S}^{1}$, shows that $H S_{1}\left(\mathcal{S}^{1}\right)$, which is homeomorphic to a 2 -sphere, would be a retract of $H S_{n}\left(\mathcal{S}^{1}\right)$. Hence, $H S_{n}\left(\mathcal{S}^{1}\right)$ would not be contractible for any positive integer $n$, compare with Corollary 3.3.

The following two theorems are used in the proof of Theorem 7.1.

Theorem 4.3. Let $X$ be a continuum and let $n$ be a positive integer. If $\chi \in H S_{n}(X) \backslash\left\{q_{X}^{n}(X), F_{X}^{n}\right\}$, then $H S_{n}(X) \backslash\left\{q_{X}^{n}(X), \chi\right\}$ is arcwise connected. 
Proof. Let $\chi^{\prime} \in H S_{n}(X) \backslash\left\{q_{X}^{n}(X), F_{X}^{n}, \chi\right\}$. We show that there is an arc joining $\chi^{\prime}$ and $F_{X}^{n}$ contained in $H S_{n}(X) \backslash\left\{q_{X}^{n}(X), \chi\right\}$. Let $A_{1}, \ldots, A_{k}$ be the components of $\left(q_{X}^{n}\right)^{-1}\left(\chi^{\prime}\right)$.

Suppose that $\left(q_{X}^{n}\right)^{-1}\left(\chi^{\prime}\right) \backslash\left(q_{X}^{n}\right)^{-1}(\chi) \neq \emptyset$. Let $a_{1} \in\left(q_{X}^{n}\right)^{-1}\left(\chi^{\prime}\right) \backslash$ $\left(q_{X}^{n}\right)^{-1}(\chi)$. Without loss of generality, we assume that $a_{1} \in A_{1}$. For each $j \in\{2, \ldots, k\}$, let $a_{j} \in A_{j}$. By [17, (1.8)], there exists an order arc $\alpha:[0,1] \rightarrow \mathcal{C}_{n}(X)$ such that $\alpha(0)=\left\{a_{1}, \ldots, a_{k}\right\}$ and $\alpha(1)=\left(q_{X}^{n}\right)^{-1}\left(\chi^{\prime}\right)$. Note that, by construction, $\left\{X,\left(q_{X}^{n}\right)^{-1}(\chi)\right\} \cap \alpha([0,1])=\emptyset$. Hence, $q_{X}^{n} \circ \alpha:[0,1] \rightarrow$ $H S_{n}(X)$ is an arc such that $q_{X}^{n} \circ \alpha(0)=F_{X}^{n}$ and $q_{X}^{n} \circ \alpha(1)=\chi^{\prime}$ and $\left\{q_{X}^{n}(X), \chi\right\} \cap\left(q_{X}^{n} \circ \alpha([0,1])\right)=\emptyset$.

Next, suppose that $\left(q_{X}^{n}\right)^{-1}\left(\chi^{\prime}\right) \subset\left(q_{X}^{n}\right)^{-1}(\chi)$. For each $j \in\{1, \ldots, k\}$, let $a_{j} \in A_{j}$. By $[17,(1.8)]$, there exists an order arc $\beta:[0,1] \rightarrow \mathcal{C}_{n}(X)$ such that $\beta(0)=\left\{a_{1}, \ldots, a_{k}\right\}$ and $\beta(1)=\left(q_{X}^{n}\right)^{-1}\left(\chi^{\prime}\right)$. Again, by construction, $\left\{X,\left(q_{X}^{n}\right)^{-1}(\chi)\right\} \cap \beta([0,1])=\emptyset$. Thus, $q_{X}^{n} \circ \beta:[0,1] \rightarrow H S_{n}(X)$ is an arc such that $q_{X}^{n} \circ \beta(0)=F_{X}^{n}, q_{X}^{n} \circ \beta(1)=\chi^{\prime}$ and $\left\{q_{X}^{n}(X), \chi\right\} \cap\left(q_{X}^{n} \circ \beta([0,1])\right)=\emptyset$.

Therefore, $H S_{n}(X) \backslash\left\{q_{X}^{n}(X), \chi\right\}$ is arcwise connected.

Theorem 4.4. Let $X$ be a continuum and let $n$ be a positive integer. If $\chi \in H S_{n}(X) \backslash\left\{F_{X}^{n}\right\}$ is such that $H S_{n}(X) \backslash\left\{F_{X}^{n}, \chi\right\}$ is not arcwise connected, then $\left(q_{X}^{n}\right)^{-1}(\chi) \in \mathcal{C}(X)$.

Proof. Suppose there exists $\chi \in H S_{n}(X) \backslash\left\{F_{X}^{n}\right\}$ such that $H S_{n}(X) \backslash$ $\left\{F_{X}^{n}, \chi\right\}$ is not arcwise connected. This implies that $\mathcal{C}_{n}(X) \backslash\left(\left\{\left(q_{X}^{n}\right)^{-1}(\chi)\right\} \cup\right.$ $\left.\mathcal{F}_{n}(X)\right)$ is not arcwise connected. Since $\mathcal{C}_{n}(X) \backslash \mathcal{F}_{n}(X)$ is arcwise connected (by $[10,6.2]$ and the fact that singletons do not arcwise disconnect $\mathcal{C}_{n}(X)$ ), we

have that $\mathcal{C}_{n}(X) \backslash\left\{\left(q_{X}^{n}\right)^{-1}(\chi)\right\}$ is not arcwise connected. Thus, $\left(q_{X}^{n}\right)^{-1}(\chi) \in$ $\mathcal{C}(X)[10,6.2]$.

\section{Contractibility}

We give a sufficient condition for a continuum to have its $n$-fold hyperspace suspensions contractible.

Theorem 5.1. Let $X$ be a continuum and let $n$ be a positive integer. If $X$ is contractible, then $H S_{n}(X)$ is contractible.

Proof. Let $R: X \times[0,1] \rightarrow X$ be such that $R(x, 0)=x$ and $R(x, 1)=q$ for every $x \in X$ and some $q \in X$. Define $G: \mathcal{C}_{n}(X) \times[0,1] \rightarrow \mathcal{C}_{n}(X)$ by $G(A, t)=R(A \times\{t\})$. Note that $G(A, 0)=A, G(A, 1)=\{q\}$ for every $A \in \mathcal{C}_{n}(X)$ and if $A \in \mathcal{F}_{n}(X)$, then $G(A, t) \in \mathcal{F}_{n}(X)$ for each $t \in[0,1]$.

Let

$$
K: H S_{n}(X) \times[0,1] \rightarrow H S_{n}(X)
$$


be given by

$$
K(\chi, t)= \begin{cases}F_{X}^{n} & \text { if } \chi=F_{X}^{n} \\ q_{X}^{n}\left(G\left(\left(q_{X}^{n}\right)^{-1}(\chi), t\right)\right) & \text { if } \chi \neq F_{X}^{n} .\end{cases}
$$

Note that $K$ is continuous by $[4,4.3$, p. 126]. Observe also that

$$
K(\chi, 0)=\chi
$$

and

$$
K(\chi, 1)=F_{X}^{n}
$$

for each $\chi \in H S_{n}(X)$. Therefore, $H S_{n}(X)$ is contractible.

We omit the proof of the following theorem because it is similar to the one given in Theorem 5.1.

Theorem 5.2. Let $X$ be a continuum and let $n$ be a positive integer. If $\mathcal{F}_{n}(X)$ is a strong deformation retract of $\mathcal{C}_{n}(X)$, then $H S_{n}(X)$ is contractible.

Question 5.1. What continua $X$ have the property that $H S_{n}(X)$ is contractible for each positive integer $n$ ?

TheOrem 5.3. If $X$ is a locally connected continuum and $n$ is a positive integer, then $H S_{n}(X) \backslash\left\{F_{X}^{n}\right\}$ is contractible.

Proof. Since $X$ is locally connected, we assume that $X$ has a convex metric $\rho([1,16])$. Without loss of generality, we assume that $\operatorname{diam}(X) \leq 1$. Let $K_{\rho}:[0,1] \times \mathcal{C}_{n}(X) \rightarrow \mathcal{C}_{n}(X)$ be given by

$$
K_{\rho}(t, A)=\{x \in X \mid \rho(x, y) \leq t \text { for some } y \in A\} .
$$

$K_{\rho}$ is continuous $[17,(0.65 .3)(\mathrm{f})], K_{\rho}(0, A)=A$ and $K_{\rho}(1, A)=X$.

Note that $K_{\rho}\left([0,1] \times \mathcal{C}_{n}(X) \backslash \mathcal{F}_{n}(X)\right) \subset \mathcal{C}_{n}(X) \backslash \mathcal{F}_{n}(X)$. Hence, $\mathcal{C}_{n}(X) \backslash$ $\mathcal{F}_{n}(X)$ is contractible. Therefore, $H S_{n}(X) \backslash\left\{F_{X}^{n}\right\}$ is contractible by Remark 2.2.

\section{Aposyndesis}

In $[12,4.4]$ it was shown that $n$-fold hyperspace suspensions are finitely aposyndetic. Next theorem extends this result to zero-dimensional aposyndesis.

A continuum $X$ is said to be zero-dimensional aposyndetic provided that for each zero-dimensional closed subset $Z$ of $X$ and each point $x \in X \backslash Z$, there exists a subcontinuum $W$ of $X$ such that $x \in \operatorname{Int}_{X}(W) \subset W \subset X \backslash Z$.

Theorem 6.1. Let $X$ be a continuum and let $n$ be a positive integer. Then $H S_{n}(X)$ is zero-dimensional aposyndetic. 
Proof. Since $H S_{n}(X)$ is locally connected at $q_{X}^{n}(X)$ and at $F_{X}^{n}$, $H S_{n}(X)$ is zero-dimensional aposyndetic at $q_{X}^{n}(X)$ and at $F_{X}^{n}$.

Let $\chi \in H S_{n}(X) \backslash\left\{q_{X}^{n}(X), F_{X}^{n}\right\}$, and let $\mathcal{Z}$ be a zero-dimensional closed subset of $H S_{n}(X)$ such that $\chi \notin \mathcal{Z}$. Since $\mathcal{Z}$ is closed and $\chi \notin \mathcal{Z}$, there exists $\varepsilon>0$ such that

$$
C l_{\mathcal{C}_{n}(X)}\left(\mathcal{V}_{\varepsilon}^{\mathcal{H}}\left(\left(q_{X}^{n}\right)^{-1}(\chi)\right)\right) \cap \mathcal{F}_{n}(X)=\emptyset
$$

and

$$
C l_{H S_{n}(X)}\left(q_{X}^{n}\left(\mathcal{V}_{\varepsilon}^{\mathcal{H}}\left(\left(q_{X}^{n}\right)^{-1}(\chi)\right)\right)\right) \cap \mathcal{Z}=\emptyset .
$$

Let $\mathcal{Z}^{\prime}=\mathcal{Z} \backslash\left\{F_{X}^{n}\right\}$. Then $\operatorname{dim}\left(\mathcal{Z}^{\prime}\right) \leq 0$ and $\mathcal{V}_{\varepsilon}^{\mathcal{H}}\left(\left(q_{X}^{n}\right)^{-1}(\chi)\right) \cap$ $\left(q_{X}^{n}\right)^{-1}\left(\mathcal{Z}^{\prime}\right)=\emptyset$. By [15, Theorem 7$]$, there exists a subcontinuum $\mathcal{M}$ of $\mathcal{C}_{n}(X)$ such that $\left(q_{X}^{n}\right)^{-1}(\chi) \in \operatorname{Int}_{\mathcal{C}_{n}(X)}(\mathcal{M})$ and $\mathcal{M} \cap\left(q_{X}^{n}\right)^{-1}\left(\mathcal{Z}^{\prime}\right)=\emptyset$. Note that $\mathcal{M}$ may be constructed in such a way that $\mathcal{F}_{n}(X) \cap \mathcal{M}=\emptyset$. Hence, $q_{X}^{n}(\mathcal{M})$ is a subcontinuum of $H S_{n}(X)$ such that $\chi \in \operatorname{Int}_{H S_{n}(X)}\left(q_{X}^{n}(\mathcal{M})\right)$ and $q_{X}^{n}(\mathcal{M}) \cap \mathcal{Z}^{\prime}=\emptyset$. Since $\mathcal{M} \cap \mathcal{F}_{n}(X)=\emptyset, F_{X}^{n} \notin q_{X}^{n}(\mathcal{M})$. Thus, $q_{X}^{n}(\mathcal{M}) \cap \mathcal{Z}=\emptyset$. Therefore, $H S_{n}(X)$ is zero-dimensional aposyndetic.

\section{Hereditarily indeComposable CONTINUA}

In $[5,8.5]$ it was shown that hereditarily indecomposable continua have unique hyperspace suspension. We prove that hereditarily indecomposable continua have unique for $n$-fold hyperspace suspensions.

Theorem 7.1. Let $X$ be a hereditarily indecomposable continuum, and let $n \geq 2$ be a positive integer. If $Y$ is a continuum such that $H S_{n}(Y)$ is homeomorphic to $H S_{n}(X)$, then $Y$ is homeomorphic to $X$.

Proof. Let $h: H S_{n}(X) \rightarrow H S_{n}(Y)$ be a homeomorphism. Observe that $h\left(\left\{q_{X}^{n}(X), F_{X}^{n}\right\}\right)=\left\{q_{Y}^{n}(Y), F_{Y}^{n}\right\}[12,3.5]$. Let $\chi \in H S_{n}(X)$ such that $\left(q_{X}^{n}\right)^{-1}(\chi) \in \mathcal{C}(X) \backslash\left(\{X\} \cup \mathcal{F}_{1}(X)\right)$. Then $\mathcal{C}_{n}(X) \backslash\left\{\left(q_{X}^{n}\right)^{-1}(\chi)\right\}$ is not arcwise connected [10,6.9]. Hence, $H S_{n}(X) \backslash\left\{F_{X}^{n}, \chi\right\}$ is not arcwise connected. Thus, since $h$ is a homeomorphism, $H S_{n}(Y) \backslash\left\{h\left(F_{X}^{n}\right), h(\chi)\right\}$ is not arcwise connected. Then, by Theorem 4.3 and the fact that $h\left(\left\{q_{X}^{n}(X), F_{X}^{n}\right\}\right)=$ $\left\{q_{Y}^{n}(Y), F_{Y}^{n}\right\}$, we have that $h\left(F_{X}^{n}\right)=F_{Y}^{n}$ and $h\left(q_{X}^{n}(X)\right)=q_{Y}^{n}(Y)$. We also have that $\left(q_{Y}^{n}\right)^{-1}(h(\chi)) \in \mathcal{C}(Y)$ by Theorem 4.4.

Hence, we have a homeomorphism

$$
\ell: \mathcal{C}_{n}(X) \backslash \mathcal{F}_{n}(X) \rightarrow \mathcal{C}_{n}(Y) \backslash \mathcal{F}_{n}(Y)
$$

\footnotetext{
${ }^{1}$ The only place in the proof of [15, Theorem 7] at which the hypothesis of the zerodimensional subset $\mathcal{Z}$ being closed is to construct an open set about the point whose closure misses $\mathcal{Z}$. To construct $\mathcal{M}$ only the fact that $\operatorname{dim}(\mathcal{Z}) \leq 0$ is used.
} 
given by

$$
\ell(A)=\left(q_{Y}^{n}\right)^{-1}\left(h\left(q_{X}^{n}(A)\right)\right) .
$$

Note that $\ell(X)=Y$ and $\ell\left(\mathcal{C}(X) \backslash \mathcal{F}_{1}(X)\right) \subset \mathcal{C}(Y) \backslash \mathcal{F}_{1}(Y)$. As in the proof of $[5,8.5],\left.\ell\right|_{\mathcal{C}(X) \backslash \mathcal{F}_{1}(X)}$ can be extended to a map $\hat{\ell}: \mathcal{C}(X) \rightarrow \mathcal{C}(Y)$ in such a way that $\hat{\ell}$ is one-to-one and $\hat{\ell}\left(\mathcal{F}_{1}(X)\right) \subset \mathcal{F}_{1}(Y)$.

Let $Z \in \mathcal{C}(Y)$ such that $\mathcal{F}_{1}(Z)=h\left(\mathcal{F}_{1}(X)\right)$. Then $\hat{\ell}(\mathcal{C}(X))=\mathcal{C}(Z)$. Since $\hat{\ell}(X)=\ell(X)=Y, Y \in \mathcal{C}(Z)$. Hence, $Z=Y$ and $\hat{\ell}\left(\mathcal{F}_{1}(X)\right)=\mathcal{F}_{1}(Y)$. Therefore, $Y$ is homeomorphic to $X$.

\section{ACKNOWLEDGEMENT.}

The author thanks the referee for the valuable suggestions.

\section{REFERENCES}

[1] R. H. Bing, Partitioning a set, Bull. Amer. Math. Soc. 55 (1949), 1101-1110.

[2] T. A. Chapman, Lectures on Hilbert cube manifolds, American Mathematical Society, Providence, 1976.

[3] D. Curtis and N. T. Nhu, Hyperspaces of finite subsets which are homeomorphic to $\aleph_{0}$-dimensional linear metric spaces, Topology Appl. 19 (1985), 251-260.

[4] J. Dugundji, Topology, Allyn and Bacon, Inc., Boston, Mass. 1966.

[5] R. Escobedo, M. de J. López and S. Macías, On the hyperspace suspension of a continuum, Topology Appl. 138 (2004), 109-124.

[6] T. Ganea, Symmetrische Potenzen topologischer Räume, Math. Nach. 11 (1954), 305-316.

[7] J. T. Goodykoontz, Jr. Some retractions and deformation retractions on $2^{X}$ and $\mathcal{C}(X)$, Topology Appl. 21 (1985), 121-133.

[8] A. Illanes and S. B. Nadler, Jr., Hyperspaces. Fundamentals and Recent Advances, Marcel Dekker, Inc., New York, 1999.

[9] M. Levin and Y. Sternfeld, The space of subcontinua of a 2-dimensional continuum is infinite-dimensional, Proc. Amer. Math. Soc. 125 (1997), 2771-2775.

[10] S. Macías, On the hyperspaces $\mathcal{C}_{n}(X)$ of a continuum X, Topology Appl. 109 (2001), 237-256.

[11] S. Macías, On the hyperspaces $\mathcal{C}_{n}(X)$ of a continuum X. II, Topology Proc. 25 (2000), Spring, 255-276.

[12] S. Macías, On the $n$-fold hyperspace suspension of continua, Topology Appl. 138 (2004), 125-138.

[13] S. Macías, Topics on Continua, Chapman \& Hall/CRC, Boca Raton, 2005.

[14] S. Macías and S. B. Nadler, Jr., Z-sets in hyperspaces, Questions Answers Gen. Topology 19 (2001), 227-241.

[15] J. M. Martínez-Montejano, Zero-dimensional closed set aposyndesis and hyperspaces, to appear in Houston J. Math.

[16] E. E. Moise, Grille decomposition and convexification theorems for compact metric locally connected continua, Bull. Amer. Math. Soc. 55 (1949), 1111-1121.

[17] S. B. Nadler, Jr., Hyperspaces of Sets. A Text with Research Questions, Marcel Dekker, Inc., New York-Basel, 1978.

[18] S. B. Nadler, Jr., A fixed point theorem for hyperspace suspensions, Houston J. Math. 5 (1979), 125-132.

[19] S. B. Nadler, Jr., Continuum Theory. An Introduction, Marcel Dekker, Inc., New York, 1992. 
[20] M. Wojdisławski, Rétractes absolus et hyperespaces des continus, Fund. Math. 32 (1939), 184-192.

S. Macías

Instituto de Matemáticas, UNAM

Circuito Exterior, Ciudad Universitaria

México D. F., C. P. 04510

Mexico

E-mail: macias@servidor.unam.mx

Received: 23.5.2005.

Revised: 30.9.2005. 